\title{
Comunicación Parlamentaria 2.0: El Debate de la Moción de Censura en Twitter
}

\author{
Marta Pérez Gabaldón| marta.perez@uchceu.es \\ Universidad CEU-Cardenal Herrera. CEU-Universities. \\ Blanca Nicasio Varea | blanca.nicasio@uchceu.es \\ Universidad CEU-Cardenal Herrera. CEU-Universities.
}

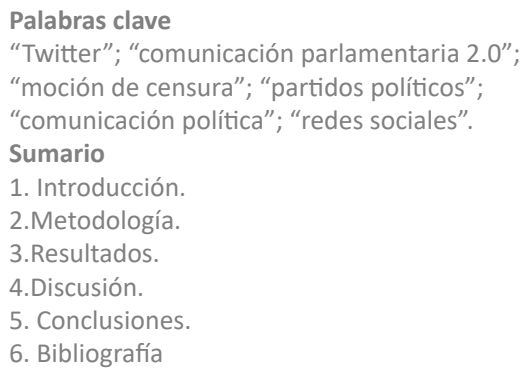

Palabras clave

"Twitter"; "comunicación parlamentaria 2.0";

"moción de censura"; "partidos políticos";

"comunicación política"; "redes sociales".

Sumario

1. Introducción.

2.Metodología.

3.Resultados.

4.Discusión.

5. Conclusiones.

6. Bibliografía

\section{Resumen}

Los días 31 de mayo y 1 de junio de 2018 tuvo lugar la cuarta moción de censura de la historia de nuestra democracia. En esta, al igual que sucedió durante la celebrada en junio de 2017, las redes sociales van a adquirir un protagonismo en la difusión y transmisión de la información, la opinión y el análisis por parte de los principales actores involucrados, así como del conjunto

de la sociedad española. Esto es así por cuanto las redes sociales son ya un instrumento consolidado en el desarrollo de la comunicación y el debate político, ya que favorecen la participación ciudadana, la comunicación instantánea y la interacción del político con los usuarios. Es por ello que la presente investigación tiene por objeto examinar los mensajes publicados en Twitter tanto por los líderes de los Partidos políticos de ámbito nacional con representación parlamentaria, como por los propios partidos. Con ello se pretende identificar, en términos cuantitativos, el impacto de los mensajes y las tendencias en la actividad de la red social durante los días del debate y, en términos cualitativos, el contenido de los mismos en base a una serie de variables preestablecidas.

\section{Cómo citar este texto:}

Marta Pérez Gabaldón, Blanca Nicasio Varea (2019): Comunicación Parlamentaria 2.0: El Debate de la Moción de Censura en Twitter, en Miguel Hernández Communication Journal, n¹0 (1), pp. 147 a 164 . Universidad Miguel Hernández, UMH (Elche-Alicante). DOI: http://dx.doi.org/10.21134/mhcj.v10i0.285 


\title{
Parliamentary Communication 2.0: The Debate on Censure Motion in Twitter
}

\author{
Marta Pérez Gabaldón| marta.perez@uchceu.es \\ Universidad CEU-Cardenal Herrera. CEU-Universities. \\ Blanca Nicasio Varea | blanca.nicasio@uchceu.es \\ Universidad CEU-Cardenal Herrera. CEU-Universities.
}

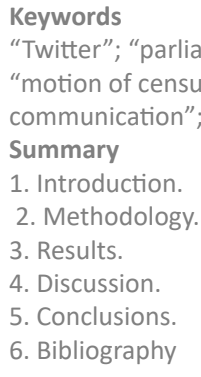

\section{Abstract}

The main objective of this paper is to perform a research in order to get to know the Miguel Trillo Archive deposited in the Library of the Museo Nacional Centro de Arte Reina Sofía (MNCARS). In addition, a review of the biography of photographer Miguel Trillo, the
\end{abstract} historic context and other concepts related to his work, such as urban tribes and a brief history of the fanzine in Spain, will be performed. A documentary analysis has been carried out following the guide of Salvador Benítez, Olivera Zaldua and Sánchez Vigil, proposing a formal analysis and a denotative and connotative analysis of content. In addition, the work is supported by a personal interview with the photographer. The most relevant conclusions corroborate the fundamental role of Miguel Trillo as a documentary photographer of the urban tribes in Madrid in the 80s of last century; but also the way his legacy has been taken by contemporary photographers such as Laura C. Vela or Davit Ruiz in their aesthetic aspects but especially in their documentary and conceptual strategy.

\section{How to cite this text:}

Marta Pérez Gabaldón, Blanca Nicasio Varea(2019): Parliamentary Communication 2.0: The Debate on Censure Motion in Twitter, in Miguel Hernández Communication Journal, n¹0 (1), pp. 147 a 164. Universidad Miguel Hernández, UMH (Elche-Alicante). DOI: http://dx.doi.org/10.21134/mhcj.v10i0.285 


\section{Introducción}

En los últimos meses, se han vivido un sinfín de acontecimientos de amplia relevancia política en nuestro país. Uno de ellos, fue la celebración de la cuarta moción de censura de la historia de nuestra democracia, los días 31 de mayo y 1 de junio de 2018. Esta es la cuarta vez que se recurre a este instrumento parlamentario en la historia de nuestra democracia, si bien es la primera vez que culmina con éxito ${ }^{1}$.

Dada la relevancia social y política de este acontecimiento nacional, la presente investigación tiene por objeto examinar los mensajes publicados en Twitter tanto por los líderes de los Partidos políticos de ámbito nacional con representación parlamentaria, como por los propios partidos durante los dos días del debate parlamentario de la moción de censura. Con ello se pretende identificar, en términos cuantitativos, el impacto de los mensajes y las tendencias en la actividad de la red social durante los días del debate y, en términos cualitativos, el contenido de los mismos en base a una serie de variables preestablecidas, sin dejar de lado la interacción con la ciudadanía. El motivo de plantear este objeto de estudio reposa sobre el hecho de que Twitter se ha convertido en un medio consolidado en la comunicación política (Campos - Domínguez, 2017: 785). Los políticos y sus respectivos partidos incorporan esta red de microblogging a sus perfiles públicos como una herramienta de comunicación y propaganda y, más específicamente, como canal de difusión inmediata de información, así como de comunicación directa y bidireccional con el usuario - ciudadano ((Túñex y Sixto, 2011). En este sentido las características propias de las nuevas tecnologías de la información y comunicación (NTICs) promueven una actitud proactiva de quienes las utilizan y constituyen condiciones y rasgos que apuntan hacia una revitalización de la esfera pública y política (Aznar Gómez, 2014: 116).

Esta plataforma, que ofrece un servicio en línea a través del cual se pueden publicar mensajes de no más de doscientos ochenta caracteres, ha sido objeto de un gran número debates y análisis académicos. Se estudia, entre otros, el papel de los emisores, de los receptores, las oportunidades de interacción, los mensajes que se difunden a través de la misma, su rol en el debate político. El gran interés en términos políticos que ha logrado despertar se debe, tal y como apuntan Arroyas, Martínez y Berná (2018: 86), a su influencia en el debate, así como a su eficacia en la creación de liderazgos, consolidación de marca política y conexión con el electorado. A lo que se podría añadir, en la misma línea, su relevante papel durante los procesos electorales, en los periodos de precampaña y campaña concretamente, así como su capacidad de movilización (García Galera, Del Hoyo Hurtado y Fernández Muñoz, 2014).

${ }^{1}$ Las tres anteriores fueron rechazadas por la mayoría parlamentaria. En los dos primeros casos (la primera fue presentada por el Grupo Parlamentario Socialista contra Adolfo Suarez en 1980, teniendo como candidato a la Presidencia de Gobierno a Felipe González; y la segunda fue presentada por el Grupo Popular en 1978 contra Felipe González, teniendo como candidato a la Presidencia del Gobierno a Antonio Hernández Mancha) cabe reseñar que se trataba de gobiernos que contaban con el respaldo de la mayoría absoluta de la cámara, lo que impedía que, dada la disciplina de partido, pudiesen salir adelante. En tiempos más recientes, en 2017, el Grupo Parlamentario Unidos Podemos presentó una nueva moción de censura, en este caso contra Mariano Rajoy, en la que se proponía como candidato a la Presidencia del Gobierno a Pablo Iglesias. En este último caso, por contraposición a lo que sucedía en los dos primeros, el Gobierno no contaba con la mayoría absoluta de la cámara, pero la formación morada no fue capaz de recabar los apoyos necesarios para que la moción pudiese salir adelante. 
Uno de los fenómenos más importantes en esta Sociedad del Conocimiento es la irrupción de las redes sociales como un complejo entramado de relaciones entre las personas. Las redes sociales son herramientas telemáticas que permiten a un usuario crear un perfil de datos sobre sí mismo en la red y compartirlo con otros usuarios. Una red social es un lugar de encuentro, de reuniones de amigos o personas con intereses comunes (Flores, 2009). Igualmente, han adquirido un gran protagonismo en la difusión y transmisión de la información, la opinión y el análisis por parte de los principales actores políticos (Cotarelo, 2013; Criado y Rojas, , así como, en general, del conjunto de la sociedad. Se podría decir, por tanto, que son ya un instrumento consolidado en el desarrollo de la comunicación y el debate político.

Por todo ello, y en coherencia con los estudios previos sobre el incremento de uso de los medios de comunicación sociales en la política (Campos-Domínguez, 2017; Zugasti y Pérez, 2015; Domingo y Martos, 2015, entre otros), y sobre la utilización de Twitter en particular (Congosto, Fernández y Moro, 2011; Moya Sánchez y Herrera Damas, 2015; Bagglionili y Rojas, 2017), se observa cómo los partidos políticos tienden a incrementar su presencia y su actividad en estos canales como instrumento de comunicación política (Domingo y Martos, 2015). En este sentido, cabe esperar un uso determinante, tanto de los líderes políticos como de sus respectivos partidos políticos, de los medios sociales, en este caso concreto de la red social Twitter, como canal de comunicación para informar sobre el procedimiento para exigir la responsabilidad política del Gobierno mediante una moción de censura (Hipótesis 1).

En esta línea, de acuerdo con estudios previos (Abejón, Sastre y Linares, 2012), se espera hallar en los perfiles de Twitter de los partidos políticos una actividad mayor respecto a sus líderes políticos, para generar y difundir contenido sobre la moción de censura (Hipótesis 2) dado que representan el canal principal y prioritario de información y comunicación sobre la actividad política no solo de la formación sino también de sus líderes. Así pues, esto sería un reflejo de la voluntad de los partidos de ocupar un espacio principal y permanente en la vida pública, frente a la itinerancia o temporalidad de los líderes, así como del interés de los mismos en centrar sus perfiles en su propia figura, más allá del proyecto común aun sin dejarlo de lado.

En cuanto a cómo utilizan las diferentes formaciones políticas estas plataformas de comunicación social, cabe subrayar que estudios previos coinciden en que los partidos y formaciones más recientes se mueven con mayor facilidad en Twitter mientras que los partidos tradicionales buscan adaptarse a esta nueva forma de comunicación, centrados más en emitir información que en comunicar y generar diálogo y conversación (Gallardo y Enguix, 2016; Fuster Fayos, 2016; Miquel-Segarra, Alonso-Muñoz, y Marcos-García, 2017). De este modo, para Gallardo y Enguix (2016: 8-9) PP y PSOE están "perdiendo la batalla" en las redes sociales respecto a los partidos más jóvenes como Podemos o Ciudadanos. De hecho, en las elecciones al Congreso de los Diputados y al Senado español que se celebraron el 20 de diciembre de 2015 los partidos Ciudadanos y Podemos utilizaron Twitter como estrategia de innovación, y lo instauraron dentro de la comunicación política como una herramienta electoral imprescindible con el fin de mostrar la política como algo cercano a la población (Carrasco, Villar, y Tejedor, 2018). Así pues, a la vista de los estudios previos, se espera hallar mayor actividad en los perfiles de los portavoces y de los partidos de más reciente creación, Podemos y Ciudadanos, respecto a los perfiles del candidato propuesto a la Presidencia del Gobierno, Pedro Sánchez, y del Presidente sometido a la moción, Mariano Rajoy, así como de sus respectivos partidos políticos durante este procedimiento recogido en la Constitución 
(Hipótesis 3).

Por otro lado, si se presta atención a la interacción con el ciudadano-usuario, atendiendo a estudios previos sobre Facebook, hallamos posiciones divergentes respecto al grado de implicación que supone el recurso al "compartir", que sería asimilable al retuit en Twitter. De este modo, por un lado, algunos autores consideran que el uso del "compartir" es la opción que mayor nivel de implicación supone para el usuario (Merino-Bobillo, LlovesSobrado y Pérez-Guerrero, 2013) y, por otro lado, otros autores situan la opción "compartir" en un nivel secundario de implicación para el usuario (Sabate, Berbegal-Mirabent, Cañabate y Lebherz, 2014; Mariani, Di Felice y Mura, 2016). Esta segunda visión es más próxima a la presente en nuestros estudios previos centrados en la red social Twitter (Pérez Gabaldón y Nicasio Varea, 2015) según los cuales los usuarios tienden a hacer uso de aquellas vías de interacción a través de las redes sociales que suponen una menor implicación, como son el "retuit" y el "me gusta", se espera hallar un mayor número de retuits y de me gusta en los mensajes publicados tanto por los partidos como por los políticos respecto al número de comentarios (Hipótesis 4). Aun y cuando el retuit y el me gusta no son las vías que mayor grado de actividad requieren por parte del usuario, no es menos cierto que su uso es una vía para evitar que la comunicación política 2.0 caiga en lo que Castells denomina "tablón de anuncios digital" (2010: 12).

En cuanto al contenido de los mensajes difundidos, teniendo en cuenta que este tipo de escenario es cada vez más empleado tanto por políticos -y sus partidos- como por los usuarios para difundir y recibir información de contenido político haciendo de este un medio consolidado en la comunicación política (Campos Domínguez, 2017: 785), se entiende que estos medios "se convierten en un escenario de confrontación política para convencer a la gran clase media de lo que debe suceder" (Domingo y Martos, 2015: 5). Así pues, se espera encontrar que la variable relativa a las críticas sea la más empleada. Si bien, en este caso específico, dado el tipo de actividad parlamentaria de la que se trata, también se espera un uso frecuente de la variable propuestas, especialmente en el perfil del candidato a la Presidencia del Gobierno y de su partido (Hipótesis 5).

\section{Metodología}

En el contexto apuntado, como se ha explicado en el epígrafe previo, en la presente investigación se analizan los mensajes publicados en Twitter por los cuatro portavoces de los Partidos políticos de ámbito nacional, que coincidentemente son aquellos con mayor representación parlamentaria, en el Congreso de los Diputados, como por sus propios partidos, durante la moción de censura, celebrada los días 31 de mayo y 1 de junio de 2018.

El objetivo consiste en examinar el uso y la gestión de la red social Twitter, concretamente, por parte de Mariano Rajoy, Presidente del Gobierno sometido a la moción, Pedro Sánchez, candidato a la Presidencia del Gobierno de España, Albert Rivera, portavoz de Ciudadanos y de Pablo Iglesias, portavoz de Podemos, así como de sus respectivos partidos. Con ello se pretende identificar, en términos cuantitativos, el impacto de los mensajes y las tendencias en la actividad de la red social durante los dos días que duró el debate y la votación y, en términos cualitativos, el contenido de los mismos en base a una serie de variables preestablecidas. Igualmente, se pretende identificar la respuesta ciudadana durante el procedimiento de esta iniciativa parlamentaria. 
Para la selección de los políticos y partidos, se optó por analizar aquellos partidos con representación parlamentaria de ámbito nacional que, tal y como se ha mencionado precedentemente, son aquellos que cuentan con un mayor número de diputados, esto es, Partido Popular, PSOE, Podemos y Ciudadanos. Respecto a la selección de los políticos, se ha optado por analizar la gestión de la red de microbbloging del Presidente sometido a la moción de censura, en el caso del PP; del candidato propuesto en la moción para la Presidencia del Gobierno, en el caso del PSOE; y a los portavoces de los Grupos Parlamentarios de Podemos y Ciudadanos. Con ello, se pretende analizar los perfiles de las figuras más relevantes, en términos políticos, de cada partido, durante el debate y votación de esta iniciativa con eficacia jurídica directa.

El estudio de los tuits se ha realizado en dos niveles, uno cuantitativo y otro cualitativo. Para el análisis cuantitativo se ha recopilado y contabilizado manualmente el número total de tuits y retuits en el período indicado ut supra. En los mensajes propios de los portavoces y de los partidos, además, se ha registrado: el número total de retuits, el número total de me gusta y el número total de comentarios.

Para el análisis cualitativo del contenido de los mensajes publicados en los perfiles de los políticos se han tenido en cuenta las siguientes variables: información relativa a la moción (fecha, normativa relativa al procedimiento, turnos de intervención, formato del debate...); anuncio de propuestas políticas; titulares de noticias publicadas en medios de comunicación sobre la moción y difusión de entrevistas en medios; promoción del trabajo desarrollado durante la legislatura; críticas a otras fuerzas políticas; y otros (en esta variable codificamos aquellos mensajes que no respondan a las anteriores). De ellas, se señala una tras la lectura completa del mensaje de manera que se trata de variables excluyentes.

Para el análisis cualitativo del contenido de los mensajes publicados en los perfiles de los partidos se han utilizado las siguientes variables: información relativa a la moción (fecha, normativa relativa al procedimiento, turnos de intervención, formato del debate...); anuncio de propuestas políticas; titulares de noticias publicadas en medios de comunicación sobre la moción y difusión de entrevistas en medios; promoción del trabajo desarrollado durante la legislatura y mensajes de apoyo a su portavoz; críticas a otras fuerzas políticas; y otros. Tal y como sucede en el análisis cualitativo de los perfiles de los líderes de los partidos, de las distintas variables, se señala una tras la lectura completa del mensaje de manera que se trata de variables excluyentes.

\section{Resultados}

Una vez recopilada y sistematizada toda la información, se ha hallado un total de 18 tuits en el perfil de Mariano Rajoy, 22 tuits tanto en el de Pedro Sánchez como en el de Albert Rivera y 24 en el de Pablo Iglesias. A su vez, se han encontrado un total de 126 tuits en la cuenta oficial del Partido Popular, 247 en la del Partido Socialista Obrero Español, 75 en la de Podemos y 209 en la de Ciudadanos.

De la comparación de los datos se desprende un uso más intenso por parte de los partidos políticos respecto de sus líderes, así como una mayor actividad por parte del partido promotor de la moción de censura. 


\section{1. Análisis cuantitativo}

Una aproximación a los resultados de la actividad de los líderes analizados en la red social, revela una tendencia de los representantes de los partidos considerados conservadores dentro del espectro político español a publicar un mayor número de mensajes propios que de retuits (15 frente a 3 en el caso de Rajoy y 13 frente a 9 en el caso de Rivera), mientras que en el caso de los representantes de los partidos de corte progresista la tendencia es la contraria, esto es, hay una mayor presencia de retuits que de tuis propios (13 frente a 9 en el caso de Sánchez y 17 frente a 7 en el caso de Iglesias). En este sentido se puede entender que el recurso al retuit es una vía a través de la cual interactuar con otros usuarios, motivo por el cual los líderes podrían optar por este recurso para asumir una posición proactiva para con sus seguidores y el resto de usuarios de la red a los que pueda llegar por este canal. No obstante, el recurso a la elaboración de tuits propios puede verse como una forma de mayor implicación directa por parte del gestor de la cuenta de usuario, tratando así de crear contenido propio para llegar a sus seguidores y que estos puedan no solo conocerlo sino difundirlo.

Gráfica 1: número tuits propios y retuits por líder político. Fuente: elaboración propia a partir de los datos obtenidos en www.twitter.es

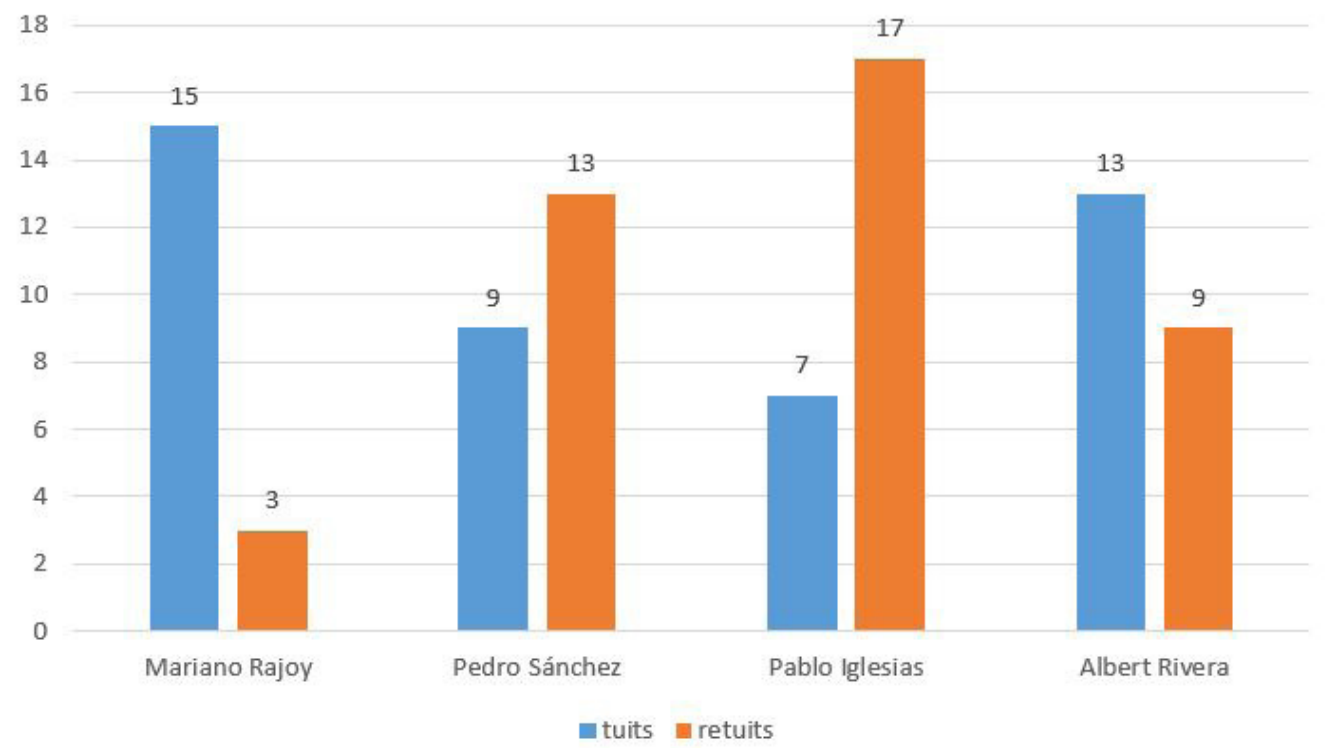

Por su parte, si se centra la atención sobre los perfiles de los partidos políticos, más allá de mayor actividad por parte de estos que por parte de los líderes a la que ya se ha hecho alusión ut supra, evidencia el interés de los mismos por elaborar y difundir mensajes propios. En este sentido, se observa cómo el 74\% (93 de 126) de los mensajes del PP, el 53\% (131 de 247) de los de PSOE, el 53\%(40 de 75), de los de Podemos y el 69\% (144 de 209) de los de Ciudadanos son de contenido propio.

Esto es así por cuanto los partidos pretenden dar promoción a sus postulados y posicionamientos a lo largo del debate, a fin de hacerlos llegar a sus seguidores y que estos puedan retuitearlos de modo que puedan tener el mayor alcance posible. Así pues, se pone de manifiesto la relevancia que Twitter ha adquirido en los últimos tiempos como canal de comunicación esencial en el ámbito político. 
Gráfica 2: número tuits propios y retuits por Partido Político. Fuente: elaboración propia a partir de los datos obtenidos en www.twitter.es

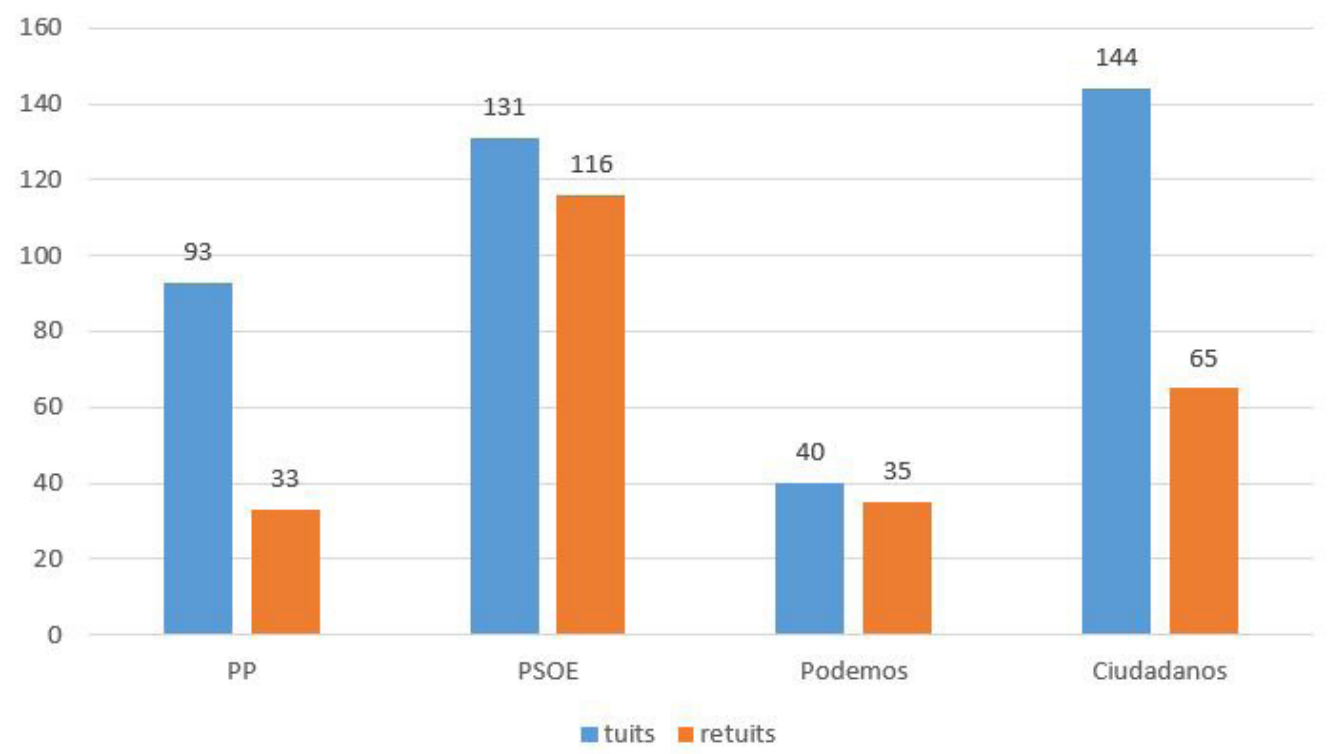

Los datos relativos a la interacción ciudadana con los líderes a partir de los tuits propios que estos publican en sus perfiles revelan, de un lado, una clara tendencia a usar prioritariamente el "me gusta" como mecanismo a través del cual los seguidores dan feedback y apoyo a los líderes.

De otro lado, el recurso menos empleado es el de la respuesta al mensaje publicado en el perfil, lo que evidencia que las opciones para interactuar con el político que menos se emplean son aquellas que requieren una mayor inversión de tiempo y esfuerzo por parte del usuario de la red.

Si centramos la atención en los números propiamente dichos, Rajoy es el que mayor número total de me gusta (71276), retuits (32236) y respuestas (15790) presenta, de modo que es perfil que mayor interacción tiene para con sus seguidores.

Sin embargo, no es el que mayor promedio de respuestas y me gusta, por cuanto en el primer caso es el perfil de Sánchez el que obtiene una media más elevada (el representante del PSOE obtiene una media de 1094 respuestas a sus publicaciones, frente a los 1050 de Rajoy) y en el segundo caso es Iglesias (el representante de Podemos obtiene una media de 5168, frente a los 4751 de Rajoy).

En cualquier caso, es significativo el elevado grado de interacción por parte de sus seguidores que obtienen a todos y cada uno de sus mensajes. 
Gráfica 3: número total de respuestas, retuits y me gusta en los perfiles de los líderes políticos. Fuente: elaboración propia a partir de los datos obtenidos en www.twitter.es

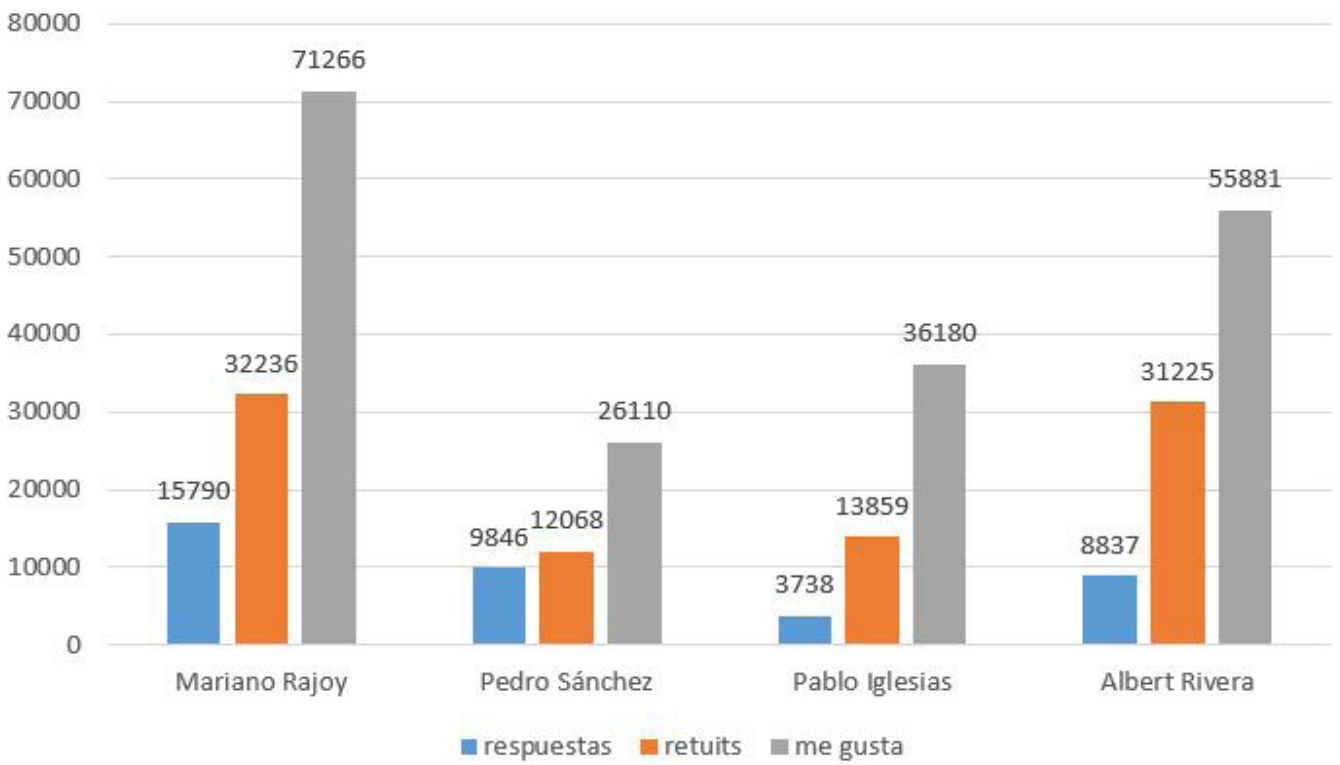

En el análisis de la interacción ciudadana con los mensajes emitidos en los perfiles de los partidos políticos, se puede observar la misma tendencia respecto a la de los perfiles de los líderes. En este sentido, el "me gusta" es el recurso más empleado por los usuarios, mientras que las respuestas son la vía de relación menos utilizada. Además, cabe destacar el escaso interés por parte de los ciudadanos a la hora de entablar una conversación con los partidos, cuestión que puede parecer lógica si se entiende que los perfiles de los líderes son de la persona, mientras estos otros son de una entidad.

Gráfica 4: número total de respuestas, retuits y me gusta en los perfiles de los Partidos políticos. Fuente: elaboración propia a partir de los datos obtenidos en www.twitter.es

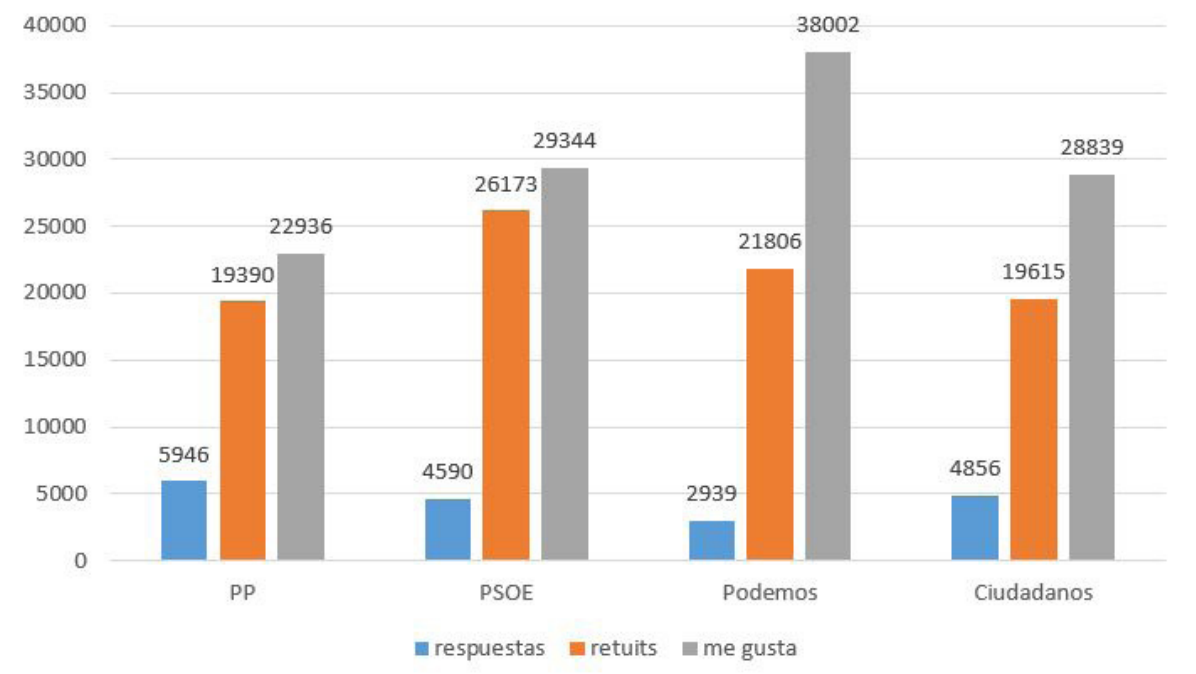


Si se atiende a los números, el Partido que más me gusta obtiene es Podemos, mientras que el PSOE obtiene mayor número total de retuits y el PP el mayor número de comentarios por parte de los seguidores.

Sin embargo, si atendemos a la media por mensaje publicado, los seguidores de la formación morada son los que más activamente emplean las tres vías existentes (73 respuestas de media, 545 retuits de media y 950 me gusta de media) para mostrar su posición respecto a lo planteado los mensajes, lo que facilita a su vez que los gestores de la cuenta del Partido puedan establecer un mayor feedback y, por ende, mostrar mayor cercanía para con el seguidor.

\section{1. Análisis cualitativo}

Una vez realizado el análisis cuantitativo, se ha procedido a clasificar los tuits propios y los retuits realizados tanto por los líderes como por los partidos políticos atendiendo a las seis variables que se han expuesto en la metodología: información sobre la moción de censura, noticias, propuestas, promoción, críticas y otros. Cada tuit se ha adscrito únicamente a una de las citadas variables atendiendo, en su caso, al contenido más relevante del mensaje ${ }^{2}$.

Dado el tipo de debate parlamentario del que se trata, no son extraños los resultados obtenidos en tanto en cuanto las variables relativas a las propuestas y las críticas a otras formaciones políticas han sido las predominantes en los perfiles de los líderes analizados.

En este sentido, en el perfil del candidato postulado a la Presidencia del Gobierno en la moción de censura constructiva se observa cómo la presentación de propuestas es la cuestión que ocupa la mayor parte de los tuits difundidos (12 de 22), realidad que se debe al papel de dicha persona dentro del propio debate que no es otro que la presentación de su programa de Gobierno.

Por su parte, en el perfil de Rajoy, Presidente al que se pretende hacer caer con la moción, las críticas hacia el candidato a la Presidencia del Gobierno son el tema central de la mayor parte de los tuits (12 de 18), haciendo especial hincapié en que el candidato pretendía ser Presidente a toda costa, sin pasar por las urnas.

De igual modo, en el perfil de Rivera también predominan las críticas, pero en este caso tanto Rajoy como hacia Sánchez, (11 de 22), especialmente al segundo de ellos, realidad que es coherente con la posición adoptada por su Grupo Parlamentario en el debate. Por último, el perfil más equilibrado es el de Iglesias en tanto en cuanto se presta una atención pareja a las propuestas (5 de 24), a la promoción (6 de 24), a la información sobre la moción (4 de 24) y a las críticas (3 de 24).

${ }^{2} \mathrm{~A}$ tal efecto, las dos investigadoras realizamos el análisis individualmente de todos los tuits que configuran la muestra del estudio. Además, en una segunda fase de control de los resultados, se seleccionó un $25 \%$ de la muestra para someterla a una nueva codificación por parte de un tercer investigador ajeno a la presente investigación, atendiendo a las variables preestablecidas. El porcentaje de coincidencia en tal fase de control superó los parámetros establecidos por Igartua para un trabajo de estas características (2006) 
Gráfica 5: Análisis cualitativo de los tuits propios en los perfiles de los líderes políticos. Fuente: elaboración propia a partir de los datos obtenidos en www.twitter.es

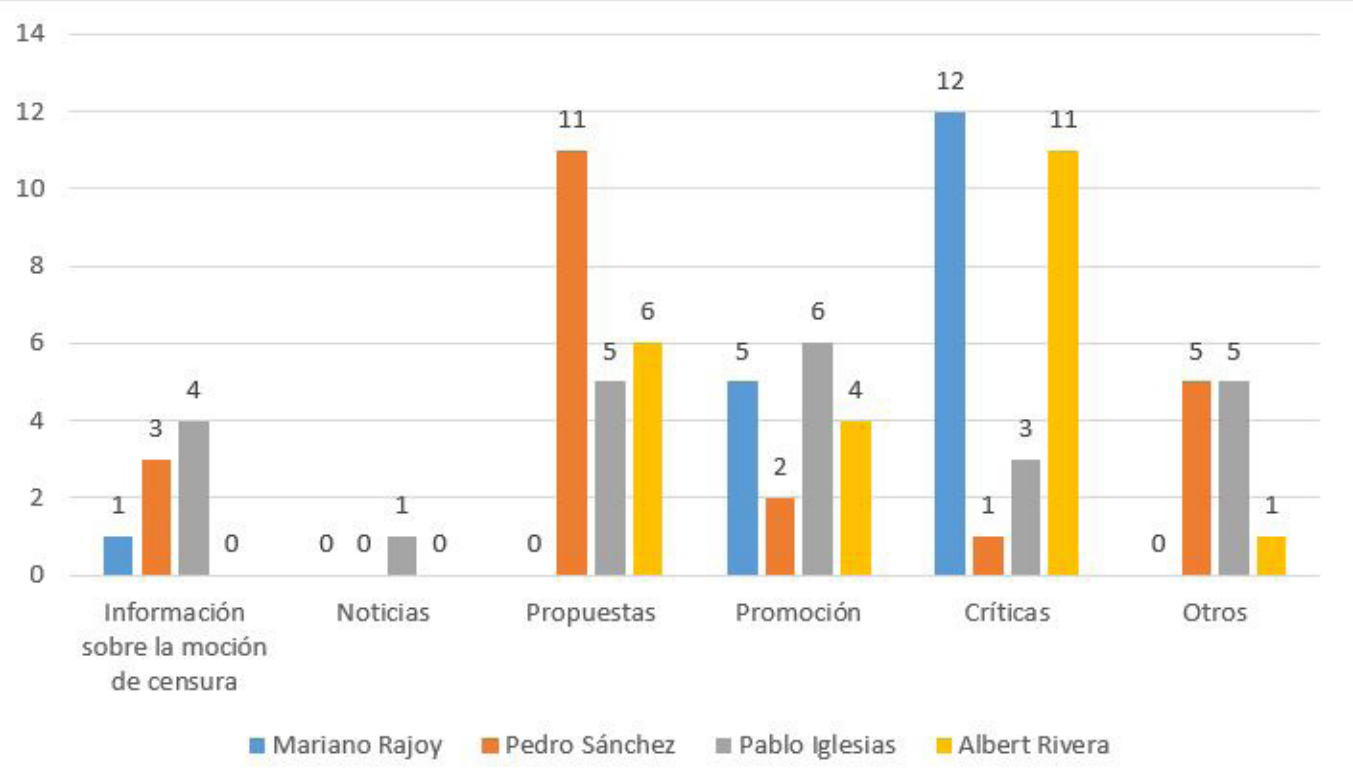

Por su parte, el análisis cualitativo de los tuits propios y los retuits publicados en los perfiles oficiales de las fuerzas políticas de ámbito nacional con representación en el Congreso de los Diputados, evidencia que la variable predominante es la crítica en todos los casos (91 de los 126 mensajes del PP, 96 de los 247 del PSOE, 26 de los 75 de Podemos y 137 de los 209 de Ciudadanos).

Cabe destacar que esta preferencia por la emisión de mensajes de carácter crítico es especialmente significativa en el caso del partido del Presidente sometido a la moción de censura (un $72 \%$ de los mensajes), haciendo referencia repetidamente los mismos, por un lado, a lo que considera el PP que es la voluntad de Sánchez de llegar a la Moncloa sin pasar por las urnas y, de otro lado, a la corrupción en el PSOE, así como también a la posición tomada por los socialistas de aceptar el respaldo de las fuerzas políticas de corte independentista con tal de garantizar el éxito de la moción promovida.

En este mismo sentido, también los perfiles de PSOE (un 38\%) y Ciudadanos (un 65\% de los mensajes) dedican buena parte de sus mensajes a lanzar críticas, si bien en el primer caso no solo se dirigen a la gestión del Gobierno Popular sino también a la posición adoptada en la moción por Ciudadanos y en el segundo caso se focalizan tanto contra el Gobierno de Rajoy como a la posición adoptada por Sánchez y los socialistas.

De este modo, el perfil más comedido a tal efecto es el de Podemos, pues las críticas ocupan un tercio de los mensajes. 
Gráfica 6: Análisis cualitativo de los tuits propios en los perfiles de los partidos políticos. Fuente: elaboración propia a partir de los datos obtenidos en www.twitter.es

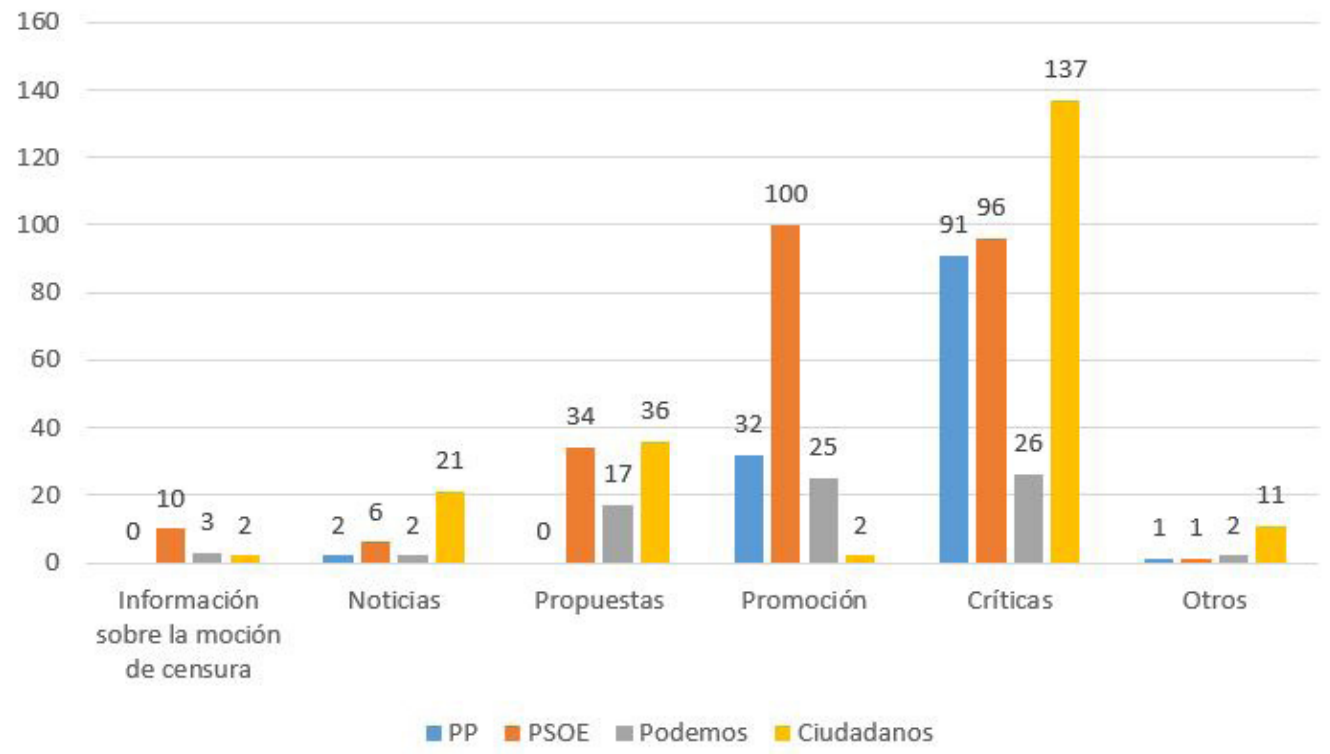

Más allá de las críticas, parece significativo destacar la escasa atención prestada por las distintas fuerzas a dar información sobre el seguimiento del debate, optando preferentemente por hacer dicho "seguimiento" recurriendo a la transcripción de las alocuciones de carácter crítico de sus respectivos portavoces, motivo por el cual tales mensajes no han sido catalogados como mensajes relativos a la información sobre la moción. De este modo, las otras dos grandes variables de relevancia son la promoción de las iniciativas y la presentación de propuestas. Respecto a la primera de ellas, cabe tener presente que se trata de la temática a la que el perfil del PSOE es el que más esfuerzo dedica al ser este el eje central de 100 de sus 247 tuits, cuestión que el Partido enlaza con el hecho de haber sido la fuerza política capaz de plantar cara a la situación y plantear la moción. Esta variable también es relevante en el $\mathrm{PP}$, cuyos tuits calificados como de promoción han sido aquellos que, en esencia, se centraban en exponer lo que el Partido considera los grandes logros de su etapa de Gobierno (32 de los 126 tuits) y en Podemos, cuyos tuits de promoción han pivotado esencialmente sobre el hecho de ser la fuerza política del cambio en tanto en cuanto son fuerza política esencial para el éxito de la moción de censura ( 25 de los 75 tuits), pero no es relevante en el caso de Ciudadanos (2 de los 209 tuits). Respecto a la variable relativa a la presentación de propuestas, cabe destacar que tema al que el PP es la única fuerza que no dedica ni uno solo de sus mensajes, cuestión que puede justificarse por el hecho de ser la fuerza política del Presidente sometido a la moción de censura, si bien sí que dedican parte de sus mensajes a esta cuestión el resto de fuerzas políticas (34 de los 247 del PSOE, 17 de los 75 de Podemos y 36 de los 209 de Ciudadanos).

\section{Discusión}

De acuerdo con los resultados expuestos en el epígrafe anterior, se ha hecho un amplio uso del entorno 2.0 analizado en el transcurso del debate de la moción de censura, los días 31 de mayo y 1 de junio. Esta afirmación, que no supone que el uso sea más intenso que en los periodos no 
analizados por cuanto excede del presente estudio realizar análisis comparativos de este tipo, confirma la hipótesis 1, en la que se prevé un uso determinante -tanto de los líderes como de sus partidos- de los medios sociales como canal de comunicación para informar sobre la moción, y pone de manifiesto el cada vez más relevante papel que las redes sociales ocupan dentro de la estrategia de comunicación política contemporánea. Este hallazgo va en la línea de los estudios previos que se planteaban como aval de la hipótesis, como es el caso de Campos-Domínguez (2017) o Domingo y Martos (2015).

Dentro de este extendido uso de la red social, la actividad por parte de los partidos políticos se produce con mayor frecuencia e intensidad a lo largo del periodo analizado respecto a la de sus figuras políticas principales, confirmándose así lo establecido en la hipótesis 2 , a partir de la cual se esperaba hallar una actividad mayor en los perfiles de los partidos respecto a sus líderes en la línea de los resultados arrojados por estudios previos (Abejón, Sastre y Linares, 2012). Si bien es cierto que a priori se podría esperar que los ciudadanos-usuarios buscasen una mayor interacción o relación directa con los líderes en tanto que representantes suyos, lo cierto es que los partidos han sabido canalizar esa voluntad de contacto con los representantes a través de los perfiles oficiales de las formaciones políticas, gracias a su elevada actividad y presencia en la red durante los días 31 de mayo y 1 de junio.

Sin embargo, aun y cuando los estudios previos apuntaban hacia un mayor uso de esta red por parte de los partidos de reciente creación y sus líderes, los datos han puesto de manifiesto que durante el debate parlamentario analizado esto no fue así plenamente. Por un lado, el partido político con un mayor número de tuits, tanto propios como retuits, es el PSOE -seguido de Ciudadanos, PP y Podemos-, por lo que en cuanto a las formaciones políticas respecta no se cumple lo planteado en la hipótesis 3. Por otro lado, en el caso de los líderes, sí se confirma lo establecido en la citada hipótesis, puesto que el representante político más activo es Pablo Iglesias, seguido de Albert Rivera, Pedro Sánchez - estos dos últimos tienen un idéntico número de mensajes totales, aunque el primero de ellos cuanta con mayor número de tuits propios-, y Mariano Rajoy, en último lugar. De este modo, se ha de considerar refutada parcialmente la Hipótesis 3, en la cual se preveía una mayor actividad en los perfiles de los portavoces y los partidos de más reciente creación.

Respecto a la interacción de la ciudadanía con los perfiles analizados, los resultados de la presente investigación demuestran que se opta, en primer lugar, por el recurso al "me gusta", en segundo lugar, por el uso del retuit y, en tercer y último lugar, por la elaboración de mensajes propios en respuesta a las publicaciones de las cuentas estudiadas. Esto no hace sino confirmar la hipótesis 4, a partir de la cual se esperaba hallar un mayor número de retuits y me gusta respecto al número de comentarios, evidenciando que el usuario tipo de esta red social tiende a emplear aquellas vías de interacción que suponen un menor esfuerzo o grado de implicación activa. No obstante, ello no resta validez a estos recursos como elementos que permiten y favorecen la comunicación bidireccional entre representantes y representados, así como entre partidos y representados. En este sentido, se observa una coincidencia plena con los resultados obtenidos en estudios previos por las autoras de la presente investigación (Pérez Gabaldón y Nicasio Varea, 2015).

Por último, y respecto al contenido propio del mensaje, cabe destacar que las variables más empleadas por parte de los líderes de los partidos han sido las críticas y las propuestas. En el caso de los perfiles de los partidos políticos, la variable más empleada ha sido la relativa a las críticas, si bien no todos los perfiles de las formaciones políticas han coincidido en la segunda 
variable más relevante: PP, PSOE y Podemos han dado preferencia a los mensajes orientados a la promoción de su actividad, mientras que Ciudadanos ha dado mayor importancia a publicar mensajes relativos a la presentación de propuestas. Así pues, a partir de esto se puede entender que se da por parcialmente confirmada la Hipótesis 5, por la que se esperaba que la variable relativa a las críticas fuese la más empleada seguida de la de propuestas. Esto es así por cuanto los ocho perfiles analizados dedican atención prioritaria a los mensajes críticos, bien hacia otros líderes bien hacia otras fuerzas políticas, pero tres de los ocho perfiles dedican más atención a la promoción que a las propuestas. A tal efecto, resulta especialmente llamativo el caso del perfil del PSOE -partido del candidato a la Presidencia del Gobierno y, por tanto, partido que avala el programa de gobierno que se está debatiendo-, por cuanto se trata de un perfil del que se esperaba que centrase mayor atención sobre las medidas planteadas en dicho programa y que optó por dar preferencia a la auto-promoción.

\section{Conclusiones}

El presente estudio avala la consolidación del uso de las redes sociales, en concreto de Twitter, como uno de los principales canales de la comunicación política y parlamentaria actual, no solo por parte de los partidos políticos sino también por parte de sus respectivos líderes. Esto es así por cuanto la citada red social permite no solo la publicación y difusión de información, sino también su uso como herramienta de posicionamiento político.

Este hecho es el que genera que, en realidades políticas como la estudiada, se encuentre un volumen de actividad significativo tanto por parte de los perfiles de los partidos políticos como por parte de los perfiles oficiales de los líderes de los mismos. Ahora bien, ha quedado demostrado que los partidos tienden a realizar una actividad más intensa, constante y conversacional, por cuanto pretenden ser los que canalicen la interacción con el ciudadano, a fin no solo de que el seguidor reciba el mensaje sino también de lograr que lo difunda, ampliando así el alcance de sus publicaciones. En este sentido, cabe destacar que si bien la tendencia habitual hasta el momento era que los partidos de reciente creación presentasen una actividad mayor y trataran de generar más diálogo y conversación con el ciudadano-usuario, el caso estudiado muestra un ligero cambio en esta tendencia al observarse cómo los partidos tradicionales también han sabido canalizar su estrategia de comunicación política y parlamentaria por esta vía de forma exitosa.

Precisamente en este último sentido, se observa cómo en el caso estudiado los usuarios recurren con frecuencia a las vías que la red social Twitter facilita para la interacción con los mensajes publicados. Sin embargo, y como ya se ha planteado en la presente investigación, los usuarios optan por hacer uso de aquellas herramientas que suponen una menor implicación y un menor esfuerzo. De este modo, el uso del me gusta y el retuit es mucho más acusado que el recurso al comentario. Si bien es cierto que de esta forma el usuario pierde la oportunidad de expresar una opinión o posicionamiento más completo y logar la interacción constante con el perfil del partido o del líder, no lo es menos que estas sencillas vías facilitan que tanto el partido como el líder puedan tener conocimiento más o menos certero de cómo llegan sus mensajes al público. Esto les puede permitir redireccionar su estrategia, en caso de ser necesario, ante el distinto seguimiento que tengan sus mensajes.

Por último, respecto al contenido de los mensajes difundidos, no cabe duda que el contexto de confrontación política se traslada al escenario 2.0. Así pues, los mensajes con contenido crítico a otros líderes o a otras formaciones políticos son los más frecuentes, o al menos uno de los 
más habituales, en todos los perfiles analizados. No obstante, en el caso que ocupa a la presente investigación, en tanto en cuanto se trata de un debate de extraordinaria relevancia y calado político como el correspondiente a una moción de censura, se ha hallado también una presencia significativa de mensajes relativos, en unos casos, a la promoción de la actividad política y, en otros casos, a la presentación de propuestas. Esto no hace sino demostrar que la realidad parlamentaria se traslada en directo a los entornos digitales con el fin de ampliar su repercusión, su alcance y su calado social, buscando siempre el feedback con los seguidores que facilitarán que esto sea así.

\section{Bibliografía}

Abejón, P., Sastre, A. y Linares, V. (2012). Facebook y Twitter en campañas electorales en España. Anuario Electrónico de Estudios en Comunicación Social Disertaciones 5 (1), pp: 130-159. Recuperado de: http://erevistas.saber.ula.ve/index.php/Disertaciones/

Arroyas, E., Martínez, H. y Berná, C. (2018). Twitter como espacio alternativo a la esfera institucional. Análisis retórico de las estrategias discursivas de Podemos durante la moción de censura contra Rajoy. Revista Mediterránea de Comunicación 11, pp. 85-94.

Aznar Gómez, H. (2014). De masas a públicos: ¿cambios hacia una democracia deliberativa?. En Aznar Gómez, H., y Pérez Llavador, J. (eds.), De la democracia de masas a la democracia deliberativa (pp. 97-126). Barcelona: Ariel.

Baggiolini, L., y Rojas, S. C. (2017). Las redes de la política: Universo narrativo, campañas y microrrelato en Twitter. Inmediaciones de la Comunicación 11(11), pp. 159-180.

Campos-Domínguez, E. (2017). Twitter y la Comunicación Política. El profesional de la información 26 (5), pp. 785-793.

Carrasco, R., Villar, E. y Tejedor, L. (2018). Twitter como herramienta de comunicación política en el contexto del referéndum independentista catalán: asociaciones ciudadanas frente a instituciones públicas. Icono 1416 (1), pp. 64-85.

Castells, M. (2010). La democràcia en l'era d'Internet'. Revista del Centre d'Estudis Jordi Pujol 12, pp. $7-13$.

Congosto, M. L., Fernández, M., \& Moro, E. (2011). Twitter y política: Información, opinión y ¿ Predicción?. Cuadernos de Comunicación Evoca 4, pp. 11-15. 
Cotarelo, R. (ed.) (2013). Ciberpolitica. Las nuevas formas de acción y comunicación políticas. Valencia: Tirant Humanidades.

Criado, J. I y Rojas Martín, F. (2013). Las redes sociales digitales en la gestión y las políticas públicas. Avances y desafíos para un gobierno abierto. Barcelona: Escola dÁdministració Pública

Domingo, J., y Martos, J. M. (2015). Análisis del discurso político en España sobre el fracaso escolar en Twitter. Archivos Analíticos de Políticas Educativas 24(70).

Flores, J.M. (2009). Nuevos modelos de comunicación, perfiles y tendencias en las redes sociales. Comunicar XVII (33), pp. 73-81.

Fuster Fayos, J. G. (2016). El uso de Twitter en comunicación politica. TFG. Universidad Jaume I, Castellón.

Gallardo Paúls, B. y Enguix Oliver, S. (2016): Pseudopolítica: el discurso político de las redes sociales. Valencia: Universitat de València.

García Galera, M.C., Del Hoyo Hurtado, M., y Fernández Muñoz, C. (2014) Jóvenes comprometidos en la Red: El papel de las redes sociales en la participación social activa. Comunicar 43, pp. 35-43.

Igartua, J. (2006). Métodos cuantitativos de investigación en comunicación. Bosch Comunicación, Barcelona.

Mariani, M. M., Di Felice, M., y Mura, M. (2016). Facebook as a destination marketing tool: Evidence from Italian regional Destination Management Organizations. Tourism Management 54, $321-343$

Merino-Bobillo, M., Lloves-Sobrado, B., Pérez-Guerrero, A.M. (2013). La interacción de los usuarios en los perfiles de Facebook de la prensa española. Palabra Clave 16 (3), pp. 842-872.

Moya Sánchez, M. y Herrera Damas, S. (2015). Cómo puede contribuir Twitter a una comunicación política más avanzada. Arbor 191(774), pp. 1-14. 
Pérez Gabaldón, M. y Nicasio Varea, B. (2015). Campañas 2.0 en la Comunidad Valenciana: las primarias en Coalició Compromís y Ciudadanos como estudio de caso. ÁMBITOS Revista Internacional de Comunicación 29, pp. 37-43.

Sabate, F., Berbegal-Mirabent, J., Cañabate, A., y Lebherz, P. R. (2014). Factors influencing popularity of branded content in Facebook fan pages. European Management Journal, 32(6), pp. 1001-1011.

Segarra, S., Muñoz, L. y García, S. (2017). Buscando la interacción. Partidos y candidatos en Twitter durante las elecciones generales de 2015. Prisma Social 18, pp. 34-54.

Túñez, M. y Sixto, J. (2011). Redes sociales, política y Compromiso 2.0: “La comunicación de los diputados españoles en Facebook. Revista Latina de Comunicación Social 66, pp. 210-246. 
MHCJ no 10 (1) | Año 2019 - Artículo no 7 (142) - Páginas 147 a 164 - mhjournal.org

\section{(c) (†)}

Licencia Creative Commons

Miguel Hernández Communication Journal

mhjournal.org

\section{Cómo citar este texto:}

Marta Pérez Gabaldón, Blanca Nicasio Varea (2019): Comunicación Parlamentaria 2.0: El Debate de la Moción de Censura en Twitter, en Miguel Hernández Communication Journal, n¹0 (1), pp. 147 a 164 . Universidad Miguel Hernández, UMH (Elche-Alicante). DOI: http://dx.doi.org/10.21134/mhcj.v10i0.285 